\title{
Precise modeling of the exoplanet host star and CoRoT main target HD 52265
}

\author{
M. E. Escobar ${ }^{1}$, S. Théado ${ }^{1}$, S. Vauclair ${ }^{1,2}$, J. Ballot ${ }^{1}$, S. Charpinet ${ }^{1}$, N. Dolez ${ }^{1}$, A. Hui-Bon-Hoa ${ }^{1}$, G. Vauclair ${ }^{1}$, \\ L. Gizon ${ }^{3,4}$, S. Mathur ${ }^{5}$, P. O. Quirion ${ }^{6}$, and T. Stahn ${ }^{4}$ \\ 1 Institut de Recherche en Astrophysique et Planétologie, Observatoire Midi-Pyrénées, CNRS, Université Paul Sabatier, \\ 14 Avenue Édouard Belin, 31400 Toulouse, France \\ e-mail: mescobar@irap.omp.eu \\ 2 Institut universitaire de France, 103 boulevard Saint Michel, 75005 Paris, France \\ 3 Max-Planck-Institut für Sonnensystemforschung, Max-Planck-Str. 2, 37191 Katlenburg-Lindau, Germany \\ ${ }^{4}$ Institut für Astrophysik, Georg-August-Universität Göttingen, Friedrich-Hundt-Platz 1, 37077 Göttingen, Germany \\ 5 High Altitude Observatory, NCAR, PO Box 3000, Boulder, CO 80307, USA \\ ${ }^{6}$ Canadian Space Agency, 6767 Boulevard de l'Aroport, Saint-Hubert, QC, J3Y 8Y9, Canada
}

Received 6 February 2012 / Accepted 23 May 2012

\section{ABSTRACT}

\begin{abstract}
Aims. This paper presents a detailed and precise study of the characteristics of the exoplanet host star and CoRoT main target HD 52265, derived from asteroseismic studies. We compare our results with previous estimates, and provide a comprehensive summary and discussion.

Methods. Our basic method is similar to that previously used by the Toulouse group for solar-type stars. Models are computed with various initial chemical compositions and the computed p-mode frequencies are compared with the observed ones. All models include atomic diffusion and we discuss the importance of radiative accelerations. Several tests are used, including the usual frequency combinations and the fits to the échelle diagrams. Possible surface effects are introduced and discussed. Automatic codes are also used to identify the best-fit model for this star (SEEK and AMP) and their results are compared with those obtained with the detailed method. Results. We find precise results for the mass, radius, and age of this star, as well as its effective temperature and luminosity. We also estimate the initial helium abundance. These results are important for the characterization of the star-planet system.
\end{abstract}

Key words. planet-star interactions - stars: interiors - stars: evolution - stars: late-type - stars: fundamental parameters asteroseismology

\section{Introduction}

In the past few years, the number of observed exoplanets has increased dramatically, owing to missions such as CoRoT (Baglin et al. 2006) and Kepler (Koch et al. 2010), as well as many other ground-based and space missions (see the exoplanet encyclopedia for a complete summary ${ }^{1}$ ). The precise characterization of exoplanet host stars becomes more and more important in the framework of the detailed studies of the observed planetary systems. Constraints on the parameters and internal structure of the star can be obtained by comparing models with photometric and spectroscopic observations (Southworth 2011; Basu et al. 2012), but the highest precision is obtained from asteroseismology, when the stellar oscillations may be observed and analyzed. This was the case, for example, for the exoplanet host stars (EHS) $\iota$ Hor (Vauclair et al. 2008) and $\mu$ Arae (Soriano \& Vauclair 2010), both observed with HARPS, as well as the EHS HAT-P-7, HAT-P-11, and TrES-2 (Christensen-Dalsgaard et al. 2010), observed with Kepler.

Among the EHS, the star HD 52265 is one of the most precisely observed for asteroseismology, as it was the only EHS observed as a main target by CoRoT. This G0V metal-rich main sequence star has an orbiting jupiter-mass planet at $0.5 \mathrm{AU}$ with a period of 119 days (Naef et al. 2001; Butler et al. 2000). It was continuously observed between December 13, 2008 and

\footnotetext{
${ }^{1}$ http://exoplanet.eu/index.php
}

March 3, 2009, that is 117 consecutive days. As a result, $31 \mathrm{p}$-mode frequencies were reported, between $1500-2550 \mu \mathrm{Hz}$, corresponding to $\ell=0,1$, and 2 (Ballot et al. 2011). From this analysis, a large separation of $\langle\Delta v\rangle=98.4 \pm 0.1 \mu \mathrm{Hz}$ and a small separation of $\left\langle\delta v_{02}\right\rangle=8.1 \pm 0.2 \mu \mathrm{Hz}$ were found, and a complete asteroseismic analysis including mode lifetimes was presented. An extensive study of the seismic rotation of HD 52265 was performed by Stahn (2011) and Gizon et al. (2012).

Spectroscopic observations of this star have been performed by several groups, who provide different values of the observed triplet $\left([\mathrm{Fe} / \mathrm{H}], \log g, T_{\text {eff }}\right)$. Their results are given in Table 1. Some of these groups also observed lines of other elements, and gave detailed relative abundances. The results show that there is an overall overmetallicity in this star, which is similar for most heavy elements, with a small dispersion. The HiPParcos parallax is $34.54 \pm 0.40$ mas (van Leeuwen 2007), which leads to a luminosity value $\log L / L_{\odot}=0.29 \pm 0.05$. A spectroscopic follow-up was also done with the Narval spectropolarimeter installed on the Bernard Lyot telescope at Pic du Midi Observatory (France) during December 2008 and January 2009, i.e., during CoRoT observations. No magnetic signature was observed.

Preliminary modeling of this star, using spectroscopic constraints, was done by Soriano et al. (2007), as preparation to CoRoT observations. Evolutionary tracks were computed using the Toulouse-Geneva evolution code (TGEC). According to the spectroscopic constraints, eight models with masses between 
Table 1. Summary of previous spectroscopic studies of HD 52265.

\begin{tabular}{lccc}
\hline \hline$[\mathrm{Fe} / \mathrm{H}]$ & $T_{\text {eff }}$ & $\log g$ & Reference \\
\hline $0.27 \pm 0.02$ & $6162 \pm 22$ & $4.29 \pm 0.04$ & Gonzalez et al. (2001) \\
$0.23 \pm 0.05$ & $6103 \pm 52$ & $4.28 \pm 0.12$ & Santos et al. (2004) \\
$0.19 \pm 0.03$ & $6069 \pm 15$ & $4.12 \pm 0.09$ & Takeda et al. (2005) \\
$0.19 \pm 0.03$ & $6076 \pm 44$ & $4.26 \pm 0.06$ & Fischer \& Valenti (2005) \\
$0.24 \pm 0.02$ & $6179 \pm 18$ & $4.36 \pm 0.03$ & Gillon \& Magain (2006) \\
$0.19 \pm 0.05$ & $6100 \pm 60$ & $4.35 \pm 0.09$ & Ballot et al. (2011) \\
\hline
\end{tabular}

$1.18 M_{\odot}$ and $1.30 M_{\odot}$ and metallicities ranging from 0.19 to 0.27 were chosen for further analysis, and adiabatic p-modes frequencies were computed. Echelle diagrams for each selected model were presented as well as their corresponding large and small separations. A large separation around $\sim 100 \mu \mathrm{Hz}$ was predicted from these models except for the Takeda et al. (2005) values, which corresponded to a smaller large separation (around $\sim 75 \mu \mathrm{Hz}$ ).

The detailed CoRoT observations allow us to progress in this analysis, using the precise seismic results. First of all, the Takeda et al. (2005) values, which correspond to a more evolved star, are excluded. We now present a complete asteroseismic analysis for HD 52265, and give precise results for the stellar parameters.

The method and models used for the asteroseismic comparisons with observations are described in Sect. 2. All the models, as described in Sect. 2.1, include element gravitational settling. The seismic tests are discussed in Sect. 2.2 and we discuss the influence of radiative accelerations on heavy elements in Sect. 2.3. The results are given in Sect. 3. Section 3.1 is devoted to the results obtained without taking surface effects into account. An analysis of surface effects and their consequences for our results is given in Sect. 3.2. A first discussion of the results is given in Sect. 3.3. Finally, in Sect. 4 the results obtained using automatic codes to find the best-fit models for this star from seismology (SEEK and AMP) are presented and compared with the previously obtained solutions. A summary and discussion are given in Sect. 5.

\section{Computations with TGEC}

\subsection{Stellar models}

Stellar models were computed using the TGEC code (Hui-Bon-Hoa 2008; Théado et al., in prep.), with the OPAL equation of state and opacities (Rogers \& Nayfonov 2002; Iglesias \& Rogers 1996), and the NACRE nuclear reaction rates (Angulo et al. 1999). Convection was treated using the mixing length theory. For all models, the mixing length parameter was adjusted to that of the solar case, i.e. $\alpha=1.8$ without either overshooting or extra-mixing. Gravitational settling of helium and metals was included using the Paquette prescription (Paquette et al. 1986; Michaud et al. 2004). Radiative accelerations of metals were also introduced using the SVP method (Single Valued Parameters approximation, see Alecian \& LeBlanc 2002; LeBlanc \& Alecian 2004; Théado et al. 2009). As most stellar evolution codes neglect these radiative accelerations, we analyzed the effects on the seismic results of introducing them or not.

Evolutionary tracks were computed for two metallicity values and two different initial helium abundances. The metallicity values were chosen as $[\mathrm{Fe} / \mathrm{H}]=0.23$ and 0.30 , so that after diffusion the final model value lies inside the observed range. Here $[\mathrm{Fe} / \mathrm{H}]$ represents the global overmetallicity with respect to the Sun, defined as $\left[\log (Z / X)_{*}-\log (Z / X)_{\odot}\right]$, where $Z$ and $X$ are computed at the stellar surface. Considering the small dispersion in the detailed abundances, this value may be compared with the observed $[\mathrm{Fe} / \mathrm{H}]$. A discussion of the computed detailed abundance variations is given in Sect. 2.3. The initial helium values are labeled $Y_{\odot}$ and $Y_{\mathrm{G}}$, where $Y_{\odot}$ is the solar helium value taken from Grevesse \& Noels (1993) and $Y_{\mathrm{G}}$ is a helium abundance that increases with $Z$ as expected if the local medium follows the general trend observed for the chemical evolution of galaxies (cf. Izotov \& Thuan 2004, 2010).

Adiabatic oscillation frequencies were computed for many models along the evolutionary tracks using the PULSE code (Brassard \& Charpinet 2008). We computed these frequencies for degrees $\ell=0$ to $\ell=3$. For comparisons with the observations (seismic tests), we used the same frequency interval for both computed and observed frequencies for consistency, i.e. between $1500 \mu \mathrm{Hz}$ and $2550 \mu \mathrm{Hz}$, as discussed below.

\subsection{Seismic tests}

A well-known characteristic of p-modes in spherical stars is that modes of the same degree with successive radial order $n$ are nearly equally spaced in frequency (e.g. Tassoul 1980). The large separation is defined as

$\Delta v_{n, \ell}=v_{n+1, \ell}-v_{n, \ell}$.

In real stars, this large separation varies slightly with frequency, so that an average value has to be used for comparisons between models and observations. One has to be careful to use the same frequency range in both cases to do the comparisons. Taking this into account, the fit between the computed and measured large separations is the first step in the process of comparisons. The large separation gives access to the stellar average density (Ulrich 1986; White et al. 2011).

A second characteristic of the $p$ modes is that the difference between $(n, \ell)$ and $(n-1, \ell+2)$ modes varies very slowly with frequency. The small separations are defined as

$\delta v_{n, \ell}=v_{n, \ell}-v_{n-1, \ell+2}$.

These small separations are most sensitive to the stellar core and may provide information on the extension of the convective core in some stars (Tassoul 1980; Roxburg \& Vorontsov 1994; Gough 1986; Soriano \& Vauclair 2008).

Provided that the stellar chemical composition is precisely known, the knowledge of both the large and the small separations, which may be plotted in the so-called C-D diagrams, gives strong constraints on the stellar parameters (ChristensenDalsgaard 1984; White et al. 2011). However, while the stellar metallicity can be derived precisely from spectroscopy, the helium content of solar-type stars cannot be directly inferred from observations. This ignorance leads to important uncertainties in the evolutionary tracks, thus in the derived stellar parameters.

We analyzed these uncertainties in detail by computing models with various chemical compositions. For each stellar evolutionary track that we computed, we first searched for the model that had an average large separation of $\langle\Delta v\rangle=98.4 \pm 0.1 \mu \mathrm{Hz}$. As the large separation continously decreases when the star evolves along the main sequence, one model only is found with the observed value (within the uncertainties). For each set of computations done with a given initial chemical composition $([\mathrm{Fe} / \mathrm{H}]$ and $Y)$, we then searched for the model that most closely fitted the small separations observed between modes of $\ell=2$ and $0,\left\langle\delta v_{02}\right\rangle=8.1 \pm 0.2 \mu \mathrm{Hz}$. We further proceed with detailed comparisons of observed and computed échelle diagrams. 


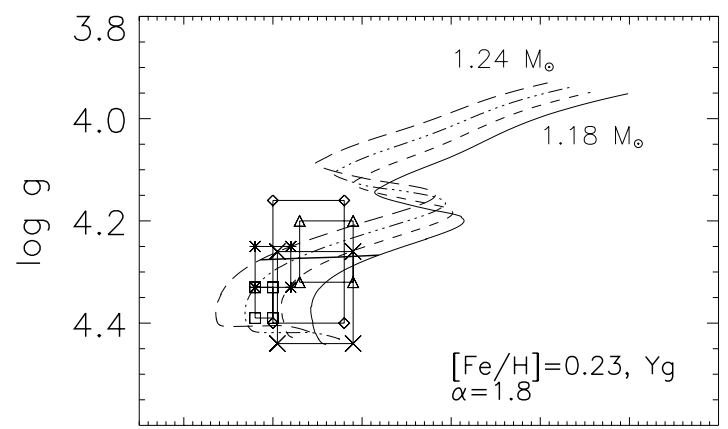

3.803 .793 .783 .773 .763 .75 $\log ($ Teff $)$

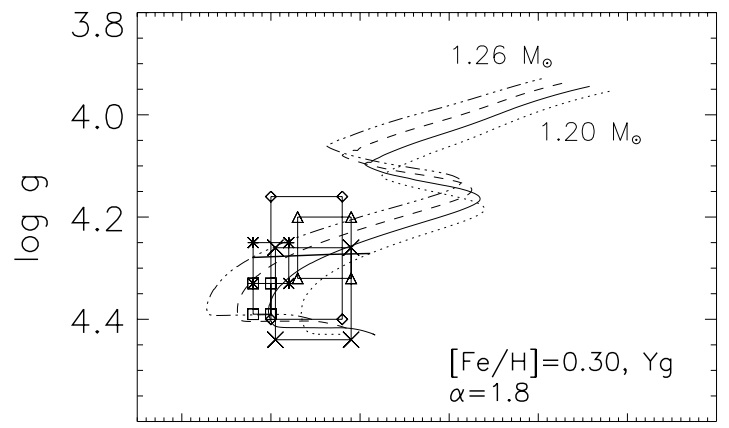

3.803 .793 .783 .773 .763 .75 $\log ($ Teff $)$

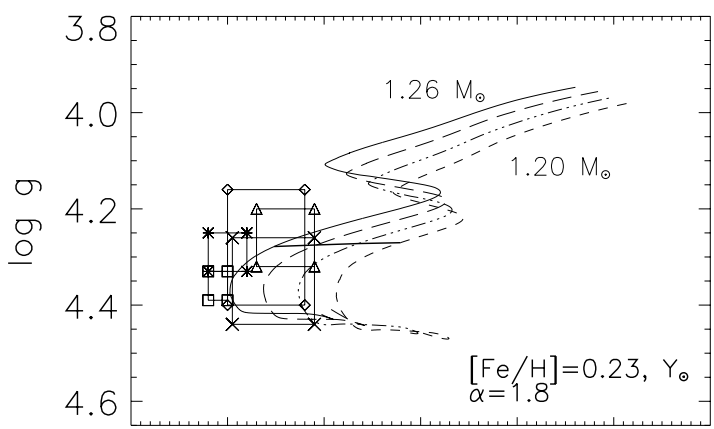

$\begin{array}{lllll}3.79 & 3.78 & 3.77 & 3.76 & 3.75\end{array}$ $\log ($ Teff)

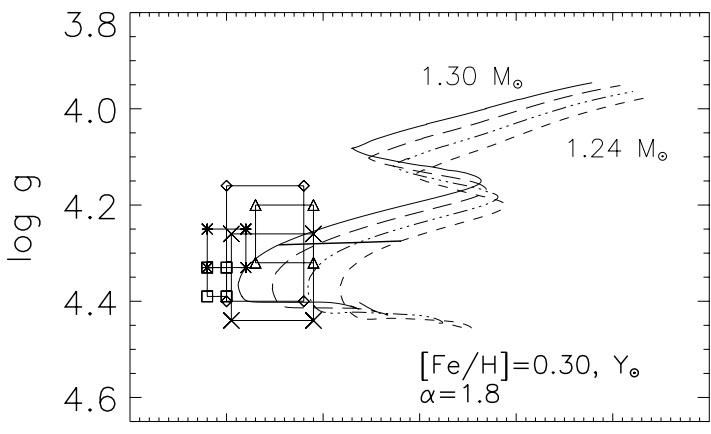

$\begin{array}{lllll}3.79 & 3.78 & 3.77 & 3.76 & 3.75\end{array}$ $\log ($ Teff)

Fig. 1. Evolutionary tracks in the $\log g$ versus $\log T_{\text {eff }}$ plane for the various sets of metallicities and helium abundance, for $\alpha=1.8$ (see text for details). The symbols indicate the error boxes of Gonzalez et al. (2001) (asterisks), Santos et al. (2004) (diamonds), Gillon \& Magain (2006) (squares), Fisher \& Valenti (2005) (triangles), and Ballot et al. (2011) (crosses). The straight thick line represents the iso- $\langle\Delta v\rangle$ line, with $\langle\Delta v\rangle=$ $98.4 \mu \mathrm{Hz}$.

In our final comparison between the models and the seismic observations, we need to take into account the surface effects induced by the wave behavior in the outer stellar layers. We computed the frequency shift induced by these effects, using the recipe proposed by Kjeldsen et al. (2008). In this case, the large separations are modified, as discussed in Sect. 3.2, which leads to corrections in the results.

\subsection{Test on atomic diffusion: radiative accelerations}

Atomic diffusion is a very important process inside stars: it can modify the atmospheric abundances and also have strong implications for the internal structure of stars (Richard et al. 2001; Michaud et al. 2004; Théado et al. 2009). At the present time, most stellar evolution codes include the computation of gravitational settling, but not the computation of radiative accelerations of heavy elements. These accelerations, which oppose gravitation, are negligible for the Sun but significant for more massive stars (Michaud et al. 1976). The variations with depth in the radiative accelerations of specific elements can lead to either their accumulation or depletion in various layers inside stars. The presence of a heavy iron layer above layers with smaller molecular weights creates an inverse $\mu$-gradient that is unstable towards thermohaline convection (Vauclair 2004). This induced mixing has also to be taken into account in computations of stellar modeling (Théado et al. 2009). An improved TGEC version including radiative accelerations on $\mathrm{C}, \mathrm{N}, \mathrm{O}, \mathrm{Ca}$, and $\mathrm{Fe}$ has been developed (Théado et al., in prep.). This new version was used to compare the oscillation frequencies computed with and without introducing the radiative accelerations in the models. For solar-type stars (masses less than $1.30 M_{\odot}$ ), we found that the difference in the computed frequencies is small. Two models with the same mass of $1.28 M_{\odot}$ and the same average large separations of $98.26 \mu \mathrm{Hz}$, one computed with the radiative accelerations and one neglecting them, display differences in the average small separations on the order of $0.01 \mu \mathrm{Hz}$. These differences between the two models decrease with decreasing stellar mass. We conclude that the radiative accelerations may be neglected in the following computations.

The result that radiative accelerations have no important consequences for the present models is consistent with the detailed abundances not showing large relative variations for the observed elements (Sect. 1). In the case of gravitational settling, the abundances of heavy elements all decrease in a similar way, in spite of their different masses, owing to the slowing down effect of the Coulomb forces. The diffusion velocities typically vary as $A / Z^{2}$ where $A$ is the mass number and $Z$ the charge, which is similar for various elements in stellar conditions. This behavior would be different if radiative accelerations were important.

\section{Results}

\subsection{Computations without surface effects}

The computations of evolutionary tracks with two initial metallicity values, $[\mathrm{Fe} / \mathrm{H}]=0.23$ and 0.30 , and two different helium abundances, the first $Y_{\odot}$ equal to 0.271 , and the second $Y_{\mathrm{G}}$ equal to 0.293 and 0.303 for the two metallicity values, leads to four different sets of tracks, each of them covering masses from $1.10 M_{\odot}$ to $1.30 M_{\odot}$. A few of these tracks are presented in Figs. 1 and 2. Error boxes of five of the spectroscopic studies given in Table 1 are also drawn in these figures. 

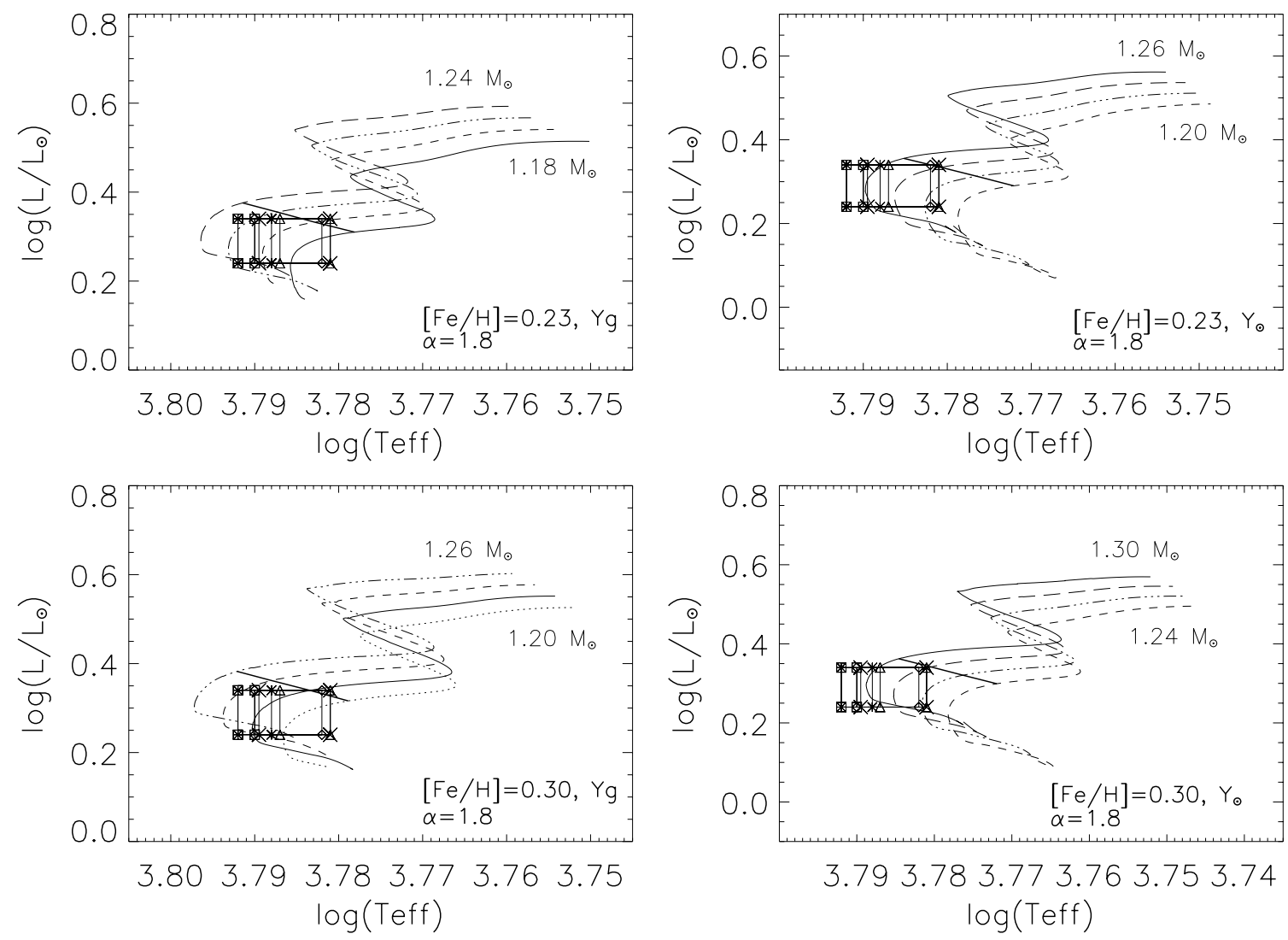

Fig. 2. HR diagrams for $\alpha=1.8$ (see text for details). Error boxes are the same horizontally as presented in Fig. 1, for the effective temperatures, and correspond vertically to the luminosity uncertainty. The straight thick line represents the iso- $\langle\Delta v\rangle$ line, with $\langle\Delta v\rangle=98.4 \mu \mathrm{Hz}$.

Table 2. Examples of models with $\alpha=1.8$, without surface effects.

\begin{tabular}{lcccccccccccc}
\hline \hline$[\mathrm{Fe} / \mathrm{H}]_{i}$ & $Y_{i}$ & $M / M_{\odot}$ & $\begin{array}{c}\text { Age } \\
{[\mathrm{Gyr}]}\end{array}$ & {$[\mathrm{Fe} / \mathrm{H}]_{\mathrm{S}}$} & $Y_{\mathrm{S}}$ & $\begin{array}{c}\log g \\
{[\mathrm{~K}]}\end{array}$ & $\log T_{\text {eff }}$ & $\log \left(L / L_{\odot}\right)$ & $R / R_{\odot}$ & $\begin{array}{c}M / R^{3} \\
{[\text { solar units }]}\end{array}$ & $\begin{array}{c}\langle\Delta v\rangle \\
{[\mu \mathrm{Hz}]}\end{array}$ & $\begin{array}{c}\left\langle\Delta v_{02}\right\rangle \\
{[\mu \mathrm{Hz}]}\end{array}$ \\
\hline 0.23 & 0.293 & 1.18 & 3.682 & 0.16 & 0.246 & 4.267 & 3.778 & 0.310 & 1.328 & 0.50 & 98.31 \\
0.23 & 0.293 & 1.20 & 3.204 & 0.16 & 0.246 & 4.271 & 3.782 & 0.332 & 1.333 & 0.51 & 98.38 & 7.54 \\
0.23 & 0.293 & 1.22 & 2.820 & 0.16 & 0.248 & 4.273 & 3.787 & 0.355 & 1.341 & 0.51 & 98.36 & 7.85 \\
0.23 & 0.293 & 1.24 & 2.416 & 0.15 & 0.242 & 4.276 & 3.791 & 0.375 & 1.347 & 0.51 & 98.34 & 8.33 \\
\hline 0.23 & 0.271 & 1.22 & 3.756 & 0.16 & 0.228 & 4.272 & 3.776 & 0.312 & 1.343 & 0.51 & 98.33 & 7.11 \\
0.23 & 0.271 & 1.24 & 3.283 & 0.16 & 0.228 & 4.275 & 3.780 & 0.334 & 1.349 & 0.51 & 98.31 & 7.80 \\
0.23 & 0.271 & 1.26 & 2.865 & 0.16 & 0.230 & 4.278 & 3.785 & 0.355 & 1.355 & 0.51 & 98.34 & 7.98 \\
0.23 & 0.271 & 1.28 & 2.461 & 0.16 & 0.227 & 4.281 & 3.789 & 0.375 & 1.361 & 0.51 & 98.35 & 8.30 \\
\hline 0.30 & 0.303 & 1.20 & 3.209 & 0.23 & 0.257 & 4.271 & 3.778 & 0.316 & 1.333 & 0.51 & 98.42 & 7.35 \\
0.30 & 0.303 & 1.22 & 2.820 & 0.23 & 0.258 & 4.273 & 3.783 & 0.339 & 1.341 & 0.51 & 98.35 & 7.75 \\
0.30 & 0.303 & 1.24 & 2.431 & 0.23 & 0.261 & 4.276 & 3.787 & 0.361 & 1.347 & 0.51 & 98.38 & 8.11 \\
0.30 & 0.303 & 1.26 & 2.072 & 0.22 & 0.254 & 4.282 & 3.792 & 0.382 & 1.354 & 0.51 & 98.38 & 8.59 \\
\hline 0.30 & 0.271 & 1.24 & 3.771 & 0.23 & 0.231 & 4.274 & 3.771 & 0.299 & 1.349 & 0.51 & 98.32 & 7.11 \\
0.30 & 0.271 & 1.26 & 3.293 & 0.23 & 0.231 & 4.277 & 3.776 & 0.320 & 1.356 & 0.51 & 98.37 & 7.64 \\
0.30 & 0.271 & 1.28 & 2.865 & 0.23 & 0.231 & 4.280 & 3.780 & 0.341 & 1.363 & 0.51 & 98.34 & 7.87 \\
0.30 & 0.271 & 1.30 & 2.476 & 0.24 & 0.233 & 4.282 & 3.784 & 0.362 & 1.369 & 0.51 & 98.39 & 8.25 \\
\hline
\end{tabular}

As explained in previous sections, we found, along each evolutionary track, a model that has an average large separation consistent with the observed one, computed in the same frequency range, of $\sim[1.5,2.5] \mathrm{mHz}$. The location of all these models in the $\log g-\log T_{\text {eff }}$ plane as well as in the HR diagrams are indicated in Figs. 1 and 2 with iso- $\langle\Delta v\rangle 98.4 \mu \mathrm{Hz}$ lines. For each case, we also computed the average small separation, $\delta v_{n, 02}$ (Table 2). We can see that for models with the same large separation, the small separation increases for increasing mass, so that in each case (i.e. for each set of chemical composition), there is a model that is consistent with both the large and small separations. However, when comparing the absolute model frequencies with the observed ones, we find that we must shift the computed frequencies by about $20 \mu \mathrm{Hz}$ to obtain the best fit to the observed ones. This offset is attributed to surface effects. The échelle diagrams corresponding to these best-fit models are given in Fig. 3.

\subsection{Computations including surface effects}

We know that stellar modeling fails to represent properly the near-surface layers of the stars. As a consequence, there is a systematic offset between the observed and computed frequencies. This offset is independent of the angular degree $\ell$ and increases 

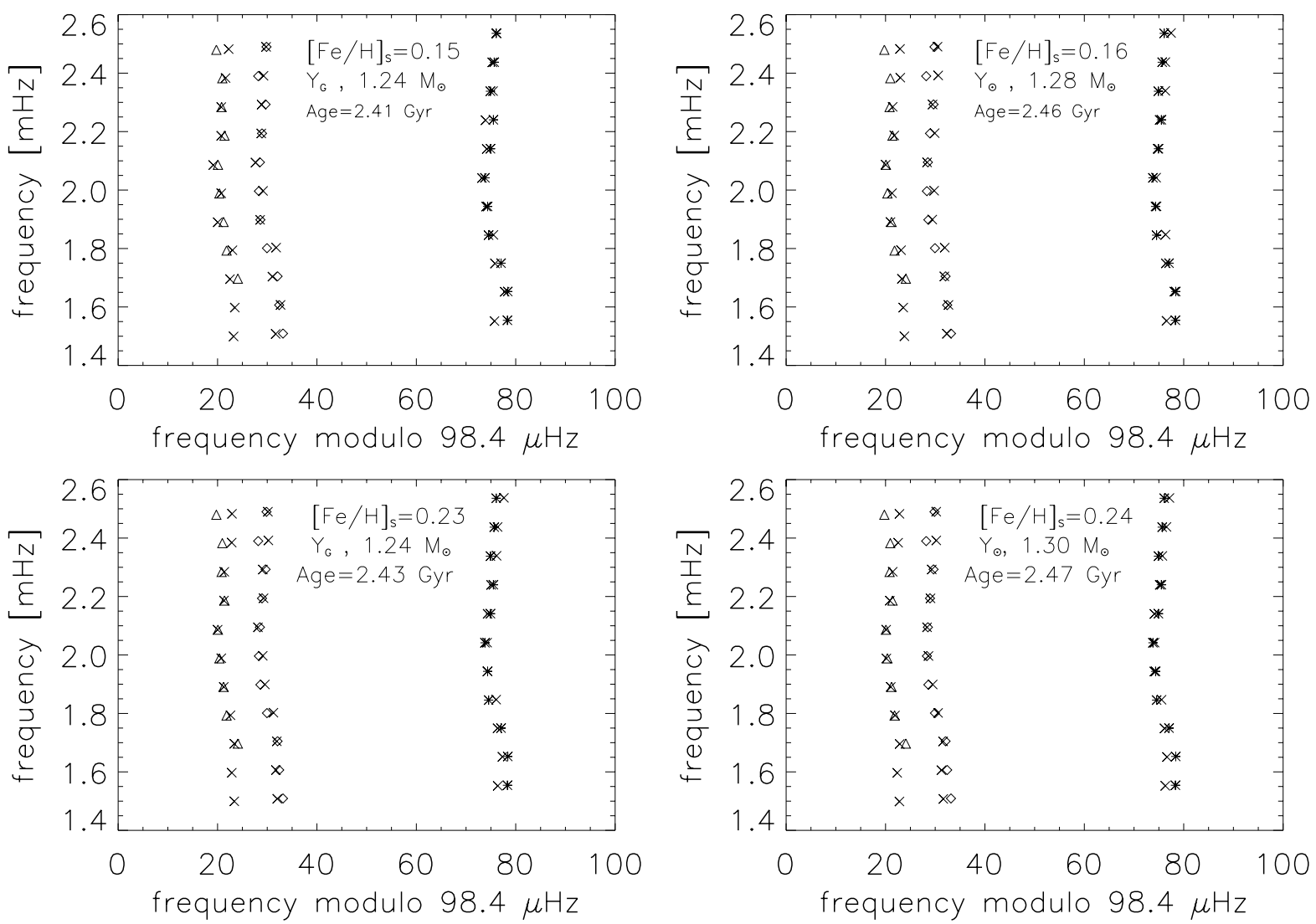

Fig. 3. Echelle diagrams for the best-fit model found for each set of evolutionary tracks calculated with $\alpha=1.8$. In this set of models, the surface effects are not included. The model frequencies (crosses) are compared to the observed frequencies, wich are represented as diamonds $(\ell=0)$, asterisks $(\ell=1)$, and triangles $(\ell=2)$. To obtain these best fits, we had to shift the model frequencies by respectively (top left, top right, bottom left, bottom right) 27, 25, 24, and $22 \mu \mathrm{Hz}$ (see text).

with frequency. This has been studied in the case of the Sun, and a similar offset is expected to occur in other stars. Using the Sun as a reference, Kjeldsen et al. (2008) suggested that for other stars, the near-surface correction to the frequencies may be approximated by

$$
v_{\mathrm{obs}}(n)-v_{\text {best }}(n)=a\left[\frac{v_{\mathrm{obs}}(n)}{v_{0}}\right]^{b} \text {, }
$$

where $v_{\mathrm{obs}}(n)$ are the observed $\ell=0$ frequencies with radial or$\operatorname{der} n, v_{\text {best }}(n)$ are the calculated frequencies for the best model, which is the model that most closely describes the star but still fails to correctly model the near-surface layers, and $v_{0}$ is a constant reference frequency chosen to be that of the frequency at the maximum amplitude in the power spectrum. The parameter $a$ may be derived as a function of the parameter $b$, which has to be adjusted to the solar case. We used this method to determine the frequency corrections that we have to apply to our models, which lead to a new average large separation $\left\langle\Delta v^{*}\right\rangle$, which is slightly larger than the observed one. We then proceed as before to derive models for the same sets of values of the helium abundance and metallicity, using $\alpha=1.8$ (Table 3). Figure 4 presents the échelle diagrams obtained with the new best-fit models. In these graphs, the frequency corrections were applied to the new model frequencies in order to compare them directly with the observations.

\subsection{Best-fit models and discussion}

We give in Table 4 the parameters of the best-fit models that we obtained for the four different sets of chemical composition.
These models have been computed with a mixing length parameter of 1.8, the frequencies have been corrected for surface effects and the computed large and small separations are the closest to the observed ones in the sample. We also give the $\chi^{2}$ values for the comparisons of the three $\ell=0,1,2$ lines in the échelle diagrams.

Figure 5 displays the large and small separations as a function of the frequency for the observations and the four bestfit models. The pattern observed in the large separations are well-reproduced by the models. For the small separations, the agreement is also very good except for two points at high frequencies $(2284 \mu \mathrm{Hz}$ and $2479 \mu \mathrm{Hz})$. This suggests that the uncertainties given in Ballot et al. (2011) for these points were underestimated.

Several concluding points can already be derived from a first analysis of Table 4. First of all, the stellar gravity is obtained, as usual, with a precision of order $0.1 \%$. The mass and age depend basically on the chosen value for the initial helium content. For a low helium value, the mass is between $1.26 M_{\odot}$ and $1.28 M_{\odot}$, and the age is $2.73 \mathrm{Gyr}$, whereas for a higher helium abundance the mass is slightly lower (around $1.22-1.23 M_{\odot}$ ) as well as the age (2.48 to $2.68 \mathrm{Gyr})$. In any case, the radius and luminosity are known with a precision of order $1 \%$.

We can go further by comparing the effective temperatures of the models with the spectroscopic observations (Fig. 6). As previously found for the cases of $\iota$ Hor (Vauclair et al. 2008) and $\mu$ Arae (Soriano \& Vauclair 2010), the effective temperatures of the best-fit models are lower for lower initial helium abundances and higher for lower metallicities. In the present case, we find that the model with the highest metallicity and the 
A\&A 543, A96 (2012)
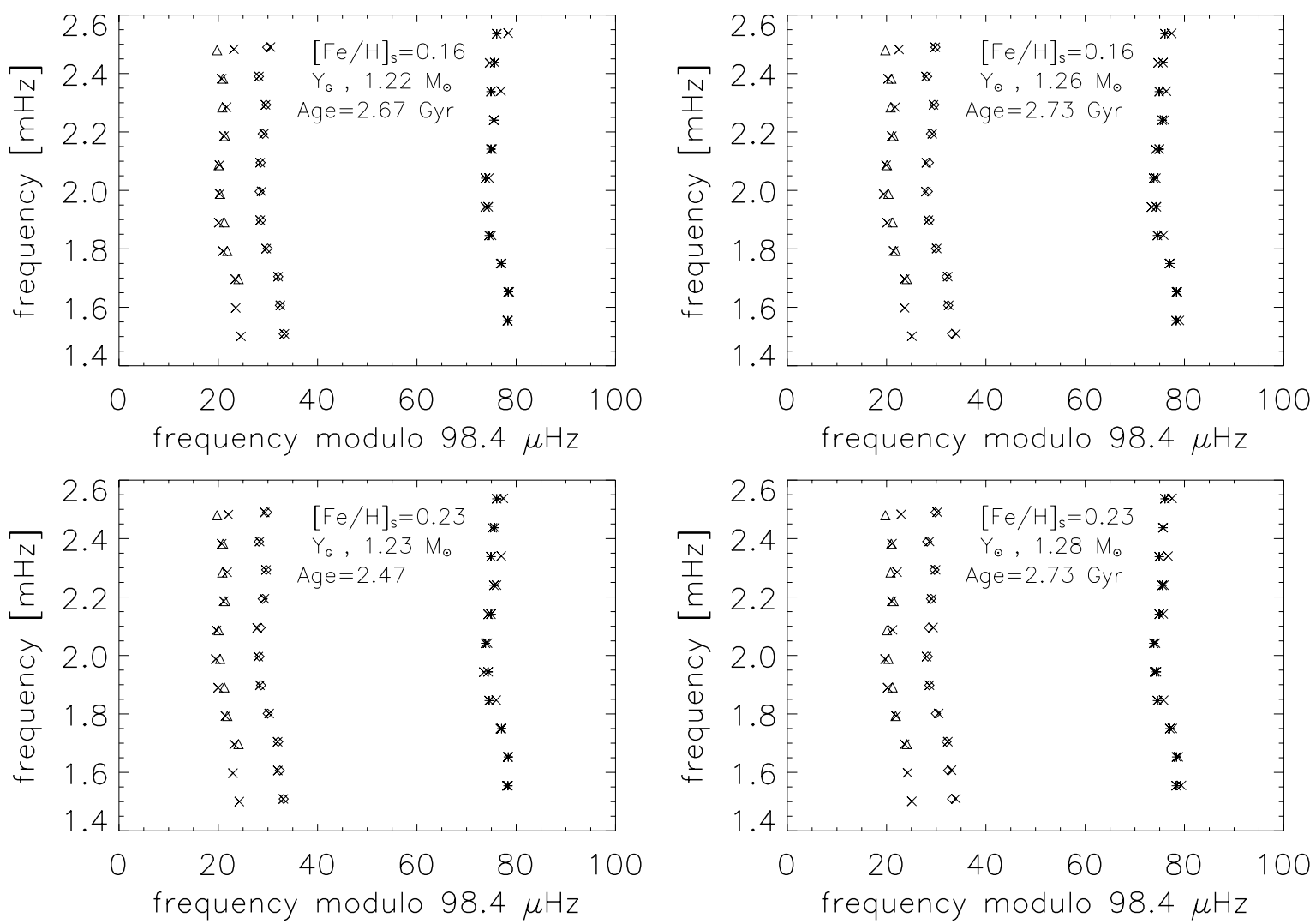

Fig. 4. Echelle diagrams for the best-fit model, including near-surface corrections, as proposed by Kjeldsen et al. (2008), found for each set of evolutionary tracks calculated with $\alpha=1.8$, in comparison with the observed frequencies. The symbols are the same as those in Fig. 3 .

Table 3. Examples of models with $\alpha=1.8$, including surface effects.

\begin{tabular}{|c|c|c|c|c|c|c|c|c|c|c|c|c|c|}
\hline$[\mathrm{Fe} / \mathrm{H}]_{i}$ & $Y_{i}$ & $M / M_{\odot}$ & $\begin{array}{l}\text { Age } \\
{[\text { Gyr] }}\end{array}$ & {$[\mathrm{Fe} / \mathrm{H}]_{\mathrm{S}}$} & $Y_{\mathrm{S}}$ & $\begin{array}{c}\log g \\
{[\mathrm{~K}]}\end{array}$ & $\log T_{\text {eff }}$ & $\log \left(L / L_{\odot}\right)$ & $R / R_{\odot}$ & $\begin{array}{c}M / R^{3} \\
\text { [solar units] }\end{array}$ & $\begin{array}{l}\left\langle\Delta v^{*}\right\rangle \\
{[\mu \mathrm{Hz}]}\end{array}$ & $\begin{array}{c}\langle\Delta v\rangle \\
{[\mu \mathrm{Hz}]}\end{array}$ & $\begin{array}{l}\left\langle\Delta v_{02}\right\rangle \\
{[\mu \mathrm{Hz}]}\end{array}$ \\
\hline 0.23 & 0.293 & 1.18 & 3.548 & 0.16 & 0.247 & 4.275 & 3.779 & 0.306 & 1.315 & 0.52 & 99.67 & 98.27 & 7.27 \\
\hline 0.23 & 0.293 & 1.20 & 3.069 & 0.16 & 0.247 & 4.279 & 3.784 & 0.328 & 1.321 & 0.52 & 99.68 & 98.27 & 7.83 \\
\hline 0.23 & 0.293 & 1.22 & 2.670 & 0.16 & 0.250 & 4.282 & 3.788 & 0.350 & 1.327 & 0.52 & 99.86 & 98.19 & 8.11 \\
\hline 0.23 & 0.293 & 1.24 & 2.251 & 0.16 & 0.244 & 4.287 & 3.792 & 0.369 & 1.331 & 0.52 & 100.08 & 98.19 & 8.47 \\
\hline 0.23 & 0.271 & 1.20 & 4.130 & 0.16 & 0.228 & 4.276 & 3.773 & 0.287 & 1.325 & 0.52 & 99.51 & 98.19 & 6.85 \\
\hline 0.23 & 0.271 & 1.22 & 3.622 & 0.16 & 0.228 & 4.279 & 3.777 & 0.309 & 1.331 & 0.52 & 99.56 & 98.18 & 7.26 \\
\hline 0.23 & 0.271 & 1.24 & 3.149 & 0.16 & 0.229 & 4.283 & 3.781 & 0.330 & 1.337 & 0.52 & 99.61 & 98.18 & 7.69 \\
\hline 0.23 & 0.271 & 1.26 & 2.730 & 0.16 & 0.232 & 4.286 & 3.786 & 0.351 & 1.343 & 0.52 & 99.62 & 98.07 & 8.11 \\
\hline 0.30 & 0.303 & 1.20 & 3.089 & 0.23 & 0.258 & 4.278 & 3.780 & 0.313 & 1.322 & 0.52 & 99.64 & 98.32 & 7.70 \\
\hline 0.30 & 0.303 & 1.22 & 2.685 & 0.23 & 0.260 & 4.282 & 3.784 & 0.335 & 1.328 & 0.52 & 99.72 & 98.13 & 7.94 \\
\hline 0.30 & 0.303 & 1.24 & 2.296 & 0.24 & 0.263 & 4.285 & 3.788 & 0.357 & 1.334 & 0.52 & 99.78 & 98.15 & 8.34 \\
\hline 0.30 & 0.303 & 1.26 & 1.907 & 0.23 & 0.257 & 4.290 & 3.793 & 0.376 & 1.337 & 0.52 & 100.23 & 98.20 & 8.84 \\
\hline 0.30 & 0.271 & 1.24 & 3.637 & 0.23 & 0.231 & 4.282 & 3.773 & 0.295 & 1.338 & 0.52 & 99.56 & 98.27 & 7.30 \\
\hline 0.30 & 0.271 & 1.26 & 3.173 & 0.23 & 0.232 & 4.284 & 3.777 & 0.317 & 1.345 & 0.52 & 99.50 & 98.16 & 7.64 \\
\hline 0.30 & 0.271 & 1.28 & 2.730 & 0.23 & 0.233 & 4.288 & 3.781 & 0.337 & 1.351 & 0.52 & 99.64 & 98.09 & 8.08 \\
\hline 0.30 & 0.271 & 1.30 & 2.326 & 0.23 & 0.236 & 4.291 & 3.785 & 0.357 & 1.355 & 0.52 & 99.80 & 98.20 & 8.52 \\
\hline
\end{tabular}

Notes. Here $\left\langle\Delta v^{*}\right\rangle$ represents the Kjeldsen et al. (2008)-corrected large separations.

lowest helium abundance, represented by black squares in Fig. 6 , is at the coolest limit of the observational boxes, thus may be excluded from the sample on spectroscopic grounds. We derive the stellar parameters from the mean value for the resulting three models, as given in Table 5.

\section{Scaling relations and automatic fits}

\subsection{Scaling relations}

The empirical scaling relations proposed by Kjeldsen \& Bedding (1995) can give approximate values of the mass and radius of a star based on the observed average large separation, the frequency at the maximum of the power spectrum, and the observed effective temperature

$$
\begin{aligned}
\frac{M}{M_{\odot}} & =\left(\frac{135 \mu \mathrm{Hz}}{\langle\Delta v\rangle}\right)^{4}\left(\frac{v_{\max }}{3050 \mu \mathrm{Hz}}\right)^{3}\left(\frac{T_{\text {eff }}}{5777 \mathrm{~K}}\right)^{3 / 2} \\
\frac{R}{R_{\odot}} & =\left(\frac{135 \mu \mathrm{Hz}}{\langle\Delta v\rangle}\right)^{2}\left(\frac{v_{\max }}{3050 \mu \mathrm{Hz}}\right)\left(\frac{T_{\text {eff }}}{5777 \mathrm{~K}}\right)^{1 / 2} .
\end{aligned}
$$

For HD 52265, the frequency at the maximum amplitude is $\nu_{\max }=2090 \pm 20 \mu \mathrm{Hz}$ (Ballot et al. 2011). With a large 
Table 4. Best-fit models obtained with the TGEC code, including near surface corrections.

\begin{tabular}{lcccccccccc}
\hline \hline$M / M_{\odot}$ & $L / L_{\odot}$ & $R / R_{\odot}$ & $\log g$ & $T_{\text {eff }}[\mathrm{K}]$ & Age $[\mathrm{Gyr}]$ & {$[\mathrm{Fe} / \mathrm{H}]_{i}$} & $Y_{i}$ & {$[\mathrm{Fe} / \mathrm{H}]_{\mathrm{S}}$} & $Y_{\mathrm{S}}$ & $\chi^{2}$ \\
\hline 1.22 & 2.239 & 1.327 & 4.282 & 6143 & 2.670 & 0.23 & 0.293 & 0.16 & 0.250 & 5.06 \\
1.23 & 2.219 & 1.330 & 4.283 & 6120 & 2.476 & 0.30 & 0.303 & 0.23 & 0.262 & 3.34 \\
1.26 & 2.244 & 1.343 & 4.286 & 6109 & 2.730 & 0.23 & 0.271 & 0.16 & 0.232 & 6.42 \\
1.28 & 2.173 & 1.351 & 4.288 & 6043 & 2.730 & 0.30 & 0.271 & 0.23 & 0.233 & 3.51 \\
\hline
\end{tabular}
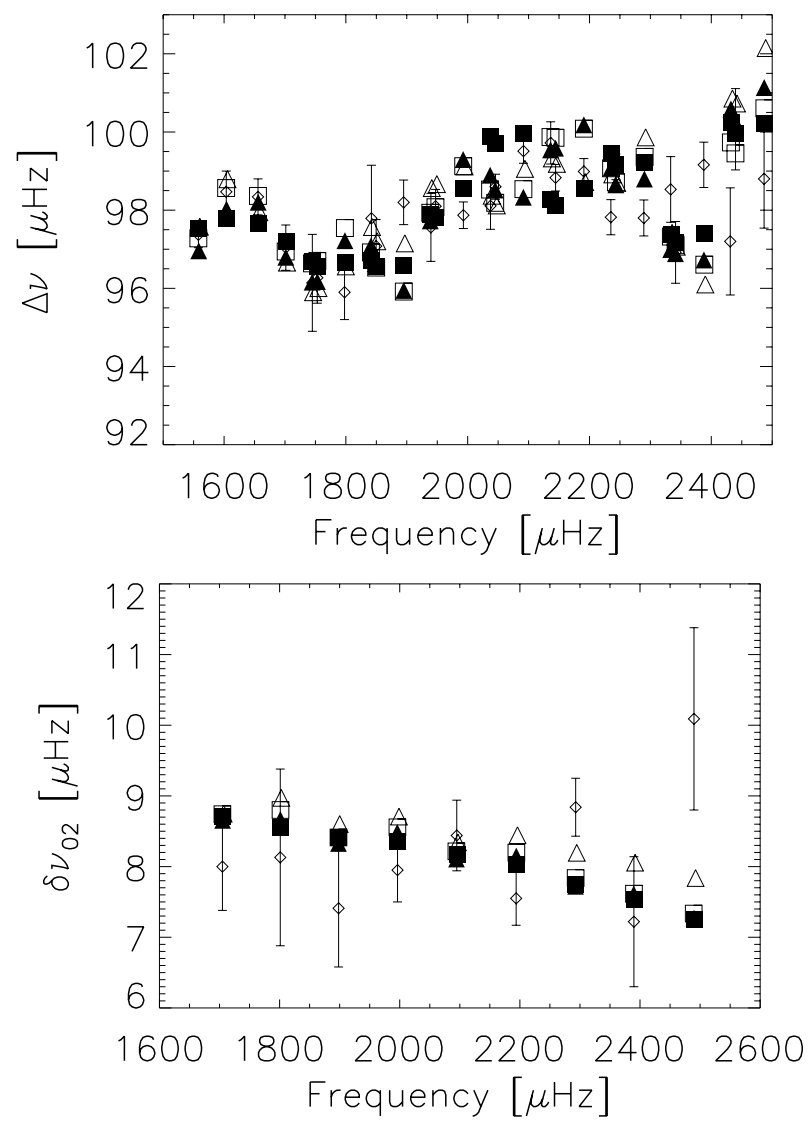

Fig. 5. Comparisons between the large separations (top) and the small separations (bottom) of the four best-fit models indicated by squares for models with $[\mathrm{Fe} / \mathrm{H}]_{\mathrm{s}}=0.23\left([\mathrm{Fe} / \mathrm{H}]_{i}=0.30\right)$ and triangles for models with $[\mathrm{Fe} / \mathrm{H}]_{\mathrm{s}}=0.16\left([\mathrm{Fe} / \mathrm{H}]_{i}=0.23\right)$. Empty symbols are used to indicate a helium abundance according to $Y_{\mathrm{g}}$, and filled symbols for a solar helium abundance (see text for details). Observations are represented as white diamonds.

separation of $98.4 \mu \mathrm{Hz}$ and an effective temperature of $6100 \mathrm{~K}$, we obtain from these relations $M=1.23 M_{\odot}$ and $R=1.32 R_{\odot}$. With a large separation corrected for the surface effects, of $99.4 \mu \mathrm{Hz}$, we obtain $M=1.19 M_{\odot}$ and $R=1.29 R_{\odot}$. In spite of the uncertainties, these results are in good agreement with our own results.

\subsection{Results from the SEEK code}

Computations have been done for this star using the SEEK automatic code (Quirion et al. 2010; Gizon et al. 2012). This code makes use of a large grid of stellar models computed with the Aarhus Stellar Evolution Code (ASTEC). It searches for the best-fit model corresponding to a seismically observed star, with the help of Bayesian statistics. The input parameters are the large and small average separations, the spectroscopic observables $\left(T_{\text {eff }}, \log g,[\mathrm{Fe} / \mathrm{H}]\right)$, and the absolute magnitude. The

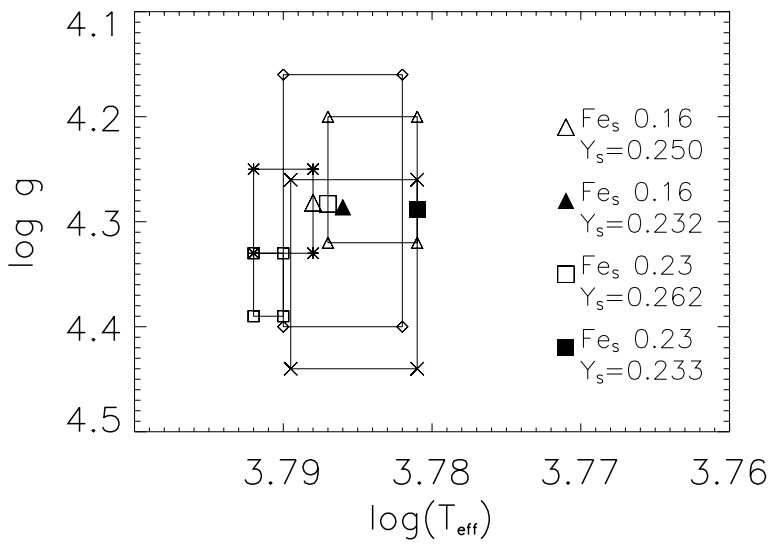

Fig. 6. Location of the best-fit models in the $\log g$ vs. $\log T_{\text {eff }}$ plane. Triangles indicate models with $[\mathrm{Fe} / \mathrm{H}]_{\mathrm{S}}=0.16\left([\mathrm{Fe} / \mathrm{H}]_{i}=0.23\right)$, and squares indicate models with $[\mathrm{Fe} / \mathrm{H}]_{\mathrm{s}}=0.23\left([\mathrm{Fe} / \mathrm{H}]_{i}=0.30\right)$. Filled symbols are used to show the models with a solar helium value. Error boxes correspond to the Gonzalez et al. (2001) (asterisks), Santos et al. (2004) (diamonds), Gillon \& Magain (2006) (squares), Fisher \& Valenti (2005) (triangles), and Ballot et al. (2011) (crosses) spectroscopic studies.

Table 5. Final results for the parameters of the exoplanet-host star HD 52265 obtained with the TGEC code.

\begin{tabular}{lc}
\hline \hline$M / M_{\odot}=1.24 \pm 0.02$ & {$[\mathrm{Fe} / \mathrm{H}]_{i}=0.27 \pm 0.04$} \\
$R / R_{\odot}=1.33 \pm 0.02$ & $Y_{i}=0.28 \pm 0.02$ \\
$L / L_{\odot}=2.23 \pm 0.03$ & {$[\mathrm{Fe} / \mathrm{H}]_{s}=0.20 \pm 0.04$} \\
$\log g=4.284 \pm 0.002$ & $Y_{\mathrm{s}}=0.25 \pm 0.02$ \\
Age $(\mathrm{Gyr})=2.6 \pm 0.2$ & $T_{\mathrm{eff}}(\mathrm{K})=6120 \pm 20$ \\
\hline
\end{tabular}

output gives the stellar mass, radius, and age. In the case of HD 52265, the values of mass and radius given by the SEEK code are slightly larger than our results, and the age is younger, i.e. $M=1.27 \pm 0.03 M_{\odot}, R=1.34 \pm 0.02 R_{\odot}$, and age $=$ $2.37 \pm 0.29$ Gyr. The differences between the SEEK results and our own may be related to a different initial helium, to slightly different values of the average large and small separations as given by Gizon et al. (2012), or to the SEEK results corresponding to a secondary maximum of probability, as discussed below.

\subsection{Results from the asteroseismic modeling portal}

We also performed computations for HD 52265 using the Asteroseismic Modeling Portal ${ }^{2}$ (AMP). The AMP provides a web-based interface for deriving the stellar parameters of Sunlike stars from asteroseismic data, and was developed at the High Altitude Observatory and the Computational \& Information Systems Laboratory of the National Center for Atmospheric Research (Metcalfe et al. 2009). It uses the ASTEC and ADIPLS codes (Christensen-Dalsgaard 2008a,b) coupled with a parallel genetic algorithm (Metcalfe \& Charbonneau 2003).

2 https://amp.ucar.edu/ 
Table 6. Final results for the parameters of the exoplanet-host star HD 52265 obtained from AMP automatic analysis, using (a) all observed frequencies or (b) only the most reliable frequencies.

\begin{tabular}{lcc}
\hline \hline & AMP(a) & AMP(b) \\
\hline$M / M_{\odot}$ & 1.22 & 1.20 \\
$R / R_{\odot}$ & 1.321 & 1.310 \\
$L / L_{\odot}$ & 2.058 & 2.128 \\
$\log g$ & 4.282 & 4.282 \\
{$[\mathrm{Fe} / \mathrm{H}]$} & 0.23 & 0.215 \\
$Y$ & 0.280 & 0.298 \\
Age $(\mathrm{Gyr})$ & 3.00 & 2.38 \\
$T_{\text {eff }}$ & 6019 & 6097 \\
\hline
\end{tabular}

Two different computations were done, the first one, $\operatorname{AMP}(a)$, by Vauclair using all the observed frequencies given by Ballot et al. (2011), and the second one, AMP(b), by S. Mathur using only the most reliable frequencies $((l=0, n=14),(l=1, n=14)$ and $(l=2, n=15)$ were excluded). The final results are very close to the parameters found by using the TGEC code (Table 6). Interestingly, the code also found solutions for a mass of $1.27 M_{\odot}$ with both a small $Y$ (about 0.26 ) and a young age (about $2.7 \mathrm{Gyr}$ ) but the $\chi^{2}$ tests showed that these results corresponded to secondary maxima, not to the best-fit solution.

\section{Summary and conclusions}

We have performed a detailed analysis of the exoplanet-host star HD 52265, which was observed by CoRoT over 117 consecutive days, as one of the main targets. The very precise observational results obtained for this star (Ballot et al. 2011) have allowed us to perform a precise determination of its parameters, using classical comparisons between models computed with the TGEC and observational data. In our computations, we have included the atomic diffusion of helium and heavy elements. For the computed stellar models, we have found that the effects of radiative accelerations on individual elements is small and may be neglected. This is consistent with the detailed abundance analysis showing similar enhancements in the heavy elements compared to the Sun. We iterated the model computations until we found a final surface metallicity $[\mathrm{Fe} / \mathrm{H}]_{\mathrm{S}}$ in the observational range. We also compared these results with those obtained using approximate scaling relations (Kjeldsen \& Bedding 1995), and automatic codes such as SEEK (Quirion et al. 2010) and AMP (Metcalfe et al. 2009). Although the detailed physics included in the models varies, these results are in good agreement.

The good concordance between results obtained with the TGEC code and the AMP for Sun-like stars had already been proven for the star $\mu$ Arae. The results for this star, which were published separately (Soriano \& Vauclair 2010, for TGEC; Doğan et al. 2012, for AMP), are presented in the Appendix for comparison. Taken together, these works represent significant advances in asteroseismic studies of Sun-like stars.

Acknowledgements. Much thanks are due to Travis Metcalfe for fruitful discussions and for introducing S.V. to A.M.P. Computational resources were provided by TeraGrid allocation TG-AST090107 through the Asteroseismic Modeling Portal (AMP). NCAR is partially supported by the Natiional Science Foundation. We also thanks the referee for important and constructive remarks.

\section{Appendix A}

Comparison of the results given by the TGEC analysis (Soriano \& Vauclair 2008) and the AMP (Doğan et al.2012) for the exoplanet-host sun-like star $\mu$ Arae.
Table A.1. Comparison of the results obtained with the TGEC and the AMP for $\mu$ Arae.

\begin{tabular}{lcc}
\hline \hline & TGEC & AMP \\
\hline$M / M_{\odot}$ & $1.1 \pm 0.02$ & 1.1 \\
$R / R_{\odot}$ & $1.36 \pm 0.06$ & 1.365 \\
$L / L_{\odot}$ & $1.90 \pm 0.10$ & 1.894 \\
$\log g$ & $4.215 \pm 0.005$ & 4.213 \\
$T_{\text {eff }}$ & $5820 \pm 50$ & 5807 \\
{$[\mathrm{Fe} / \mathrm{H}]$} & $+0.32 \pm 0.02$ & +0.418 \\
$Y$ & $0.30 \pm 0.01$ & 0.316 \\
Age $(\mathrm{Gyr})$ & $6.34 \pm 0.80$ & 6.66 \\
\hline
\end{tabular}

\section{References}

Alecian, G., \& LeBlanc, F. 2002, MNRAS, 332, 891

Angulo, C., Arnould, M., Rayer, M., et al. 1999, Nucl. Phys. A, 656, 3 , http://pntpm.ylb.ac.be/Nacre/nacre.htm

Baglin, A., Auvergne, M., Barge, P., et al. 2006, in The CoRoT Mission: Pre-launch Status, eds. M. Fridlund, A. Baglin, J. Lochard, \& L. Conroy (Noordwijk: ESA Publication Divisions), ESA SP, 1306, 33

Basu, S., Verner, G. A., Chaplin, W. J., \& Elsworth, Y. 2012, ApJ, 746, 76

Ballot, J., Gizon, L., Samadi, R., et al. 2011, A\&A, 530, 97

Brassard, P., \& Charpinet, S. 2008, Ap\&SS, 316, 107

Butler, R. P., Vogt, S., Marcy, G., et al. 2000, ApJ, 545, 504

Christensen-Dalsgaard, J. 1984, in Space Research in Stellar and Variability, eds. A. Mangeney, \& F. Praderie, 11

Christensen-Dalsgaard, J. 2008a, Ap\&SS, 316, 13

Christensen-Dalsgaard, J. 2008b, Ap\&SS, 316, 113

Christensen-Dalsgaard, J., Kjeldsen, H., Brown, T. M., et al. 2010, ApJ, 713, L164

Doğan, G., Metcalfe, T. S., Woitaszek, M., et al. 2012, ApJ, submitted

Fischer, D. A., \& Valenti, J. A. 2005, ApJ, 622, 1102

Gillon, M., \& Magain, P. 2006, A\&A, 448, 341

Gizon, L., Ballot, J., Michel, E., et al. 2012, Nature, submitted

Gonzalez, G., Laws, C., Tyagi, S., \& Reddy, B. E. 2001, ApJ, 121, 432

Gough, D. O. 1986, in Hydrodynamic and magnetohydrodynamic problems in the Sun and stars, ed. Y. Osaki (Univ. of Tokyo Press), 117

Grevesse, N., \& Noels, A. 1993, in Origin and Evolution of the Elements, eds. N. Prantzos, E. Vangioni-Flam, \& M. Cassé (Cambridge University Press), 15 Hui-Bon-Hoa, A. 2008, Ap\&SS, 316, 55

Iglesias, C. A., \& Rogers, F. J. 1996, ApJ, 464, 943

Izotov, Y. I., \& Thuan, T. X. 2004, ApJ, 602, 200

Izotov, Y. I., \& Thuan, T. X. 2010, ApJ, 710, L67

Kjeldsen, H., \& Bedding, T. R. 1995, A\&A, 293, 87

Kjeldsen, H., Bedding, T. R., \& Christensen-Dalsgaard, J. 2008, ApJ, 683, L175

Koch, D. G., Borucki, W. J., Basri, G., et al. 2010, ApJ, 713, L79

LeBlanc, F., \& Alecian, G. 2004, MNRAS, 352, 1329

van Leeuwen, F. 2007, A\&A, 474, 653

Metcalfe, T., \& Charbonneau, P. 2003, JCoPh, 185, 176

Metcalfe, T., Creevey, O. L., \& Christensen-Dalsgaard, J. 2009, ApJ, 699, 373

Michaud, G., Charland, Y., Vauclair, S., \& Vauclair, G. 1976, ApJ, 210, 447

Michaud, G., Richard, O., Richer, J., \& VandenBerg, D. 2004, ApJ, 606, 452

Naef, D., Mayor, M., Pepe, F., et al. 2001, A\&A, 375, 205

Paquette, C., Pelletier, C., Fontaine, G., \& Michaud, G. 1986, ApJS, 61, 177

Quirion, P. O., Christensen-Dalsgaard, J., \& Arenthoft, T. 2010, ApJ, 725, 2176

Richard, O., Michaud, G., \& Richer, J. 2001, ApJ, 558, 377

Rogers, F. J., \& Nayfonov, A. 2002, ApJ, 576, 1064

Roxburgh, I. W., \& Vorontsov, S. V. 1994, MNRAS, 267, 297

Santos, N. C., Israelian, G., \& Mayor, M. 2004, A\&A, 415, 1153

Soriano, M., \& Vauclair, S. 2008, A\&A, 488, 975

Soriano, M., \& Vauclair, S. 2010, A\&A, 513, A49

Soriano, M., Vauclair, S., Vauclair, G., \& Laymand, M. 2007, A\&A, 471, 885

Southworth, J. 2011, MNRAS, 417, 216

Stahn, T. 2011, Ph.D. Thesis, University of Göttingen, Germany

Takeda, Y., Ohkubo, M., Sato, B., Kambe, E., \& Sadakane, K. 2005, PASJ, 57, 27

Tassoul, M. 1980, ApJS, 43, 469

Théado, S., Vauclair, S., Alecian, G., \& LeBlanc, F. 2009, ApJ, 704, 1262

Ulrich, R. K. 1986, ApJ, 306, L37

Vauclair, S. 2004, ApJ, 605, 874

Vauclair, S., Laymand, M., Bouchy, F., et al. 2008, A\&A, 482, L5

White, T. R., Bedding, T. R., Stello, D., et al. 2011, ApJ, 743, 161 